\title{
Antiangiogenic therapy of breast cancer. How did we get here? The road not taken
}

\author{
Emilio Alba
}

$\mathrm{T}$ herapeutic strategies aimed at blocking the impaired cell signalling pathways in tumour cells have become a mainstream dogma in both cancer research and treatment today. Generically speaking, this approach has been defined as a personalised therapy for cancer, the first instance of spectacular success being treatment with imatinib for chronic myeloid leukaemia. This approach has gained a certain degree of success in breast cancer too. For instance, major advances were achieved in treating breast cancer with tamoxifen, particularly in the adjuvant setting, in patients with positive hormone receptor status and with trastuzumab in HER 2+ patients. Indeed, this is one of the main reasons underpinning the gradual decrease in mortality seen over the past few years in this kind of cancer.

Along the same line of thinking is the use of antiangiogenic drugs to treat multiple kinds of cancer, in general, and breast cancer, in particular. Anti-angiogenic drugs, such as bevacizumab (a monoclonal antibody that blocks VEGF) and various tyrosine kinase inhibitors (TKI) involved in signalling the neo-angiogenesis process, have been widely studied in the context of metastatic breast cancer. Combinations of bevacizumab in different chemotherapy regimens have been studied in five major randomised trials in disseminated breast cancer [1]. Results with the addition of bevacizumab to the chemotherapy regimen have been tremendously consistent. The clinical response rate has been seen to increase, with a modest increase in progression-free survival (PFS) at around 2 months in most studies, albeit with no impact on survival. The outcomes seen with TKIs (sunitinib, sorafenib and axitinib), either in single-drug therapy or combined with chemotherapy, have been less spectacular. These discreet outcomes, together with the toxicity associated with these drugs and their cost, have opened up major debate on their use amongst health-

\section{E. Alba (两)}

Servicio de Oncología Médica

Hospital Universitario Virgen de la Victoria

Campus de Teatinos, $\mathrm{s} / \mathrm{n}$

ES-29010 Málaga, Spain

e-mail: ealbac@uma.es care professionals, regulatory agencies and health authorities.

The time has now come, therefore, to look into the cause of these outcomes and, in particular, to address how outcomes can be enhanced through a new conceptual framework and improved experimental designs. In this issue of Clinical and Translational Oncology, Trinh et al. make a major contribution that, although based solely on clinical and molecular descriptions of two cases, sheds some light on current status [2]. The authors describe two cases of disseminated breast cancer where disease progression during therapy with bevacizumab continued to be angiogenesis dependent. This description, taken directly from clinical practice, agrees with previous data derived from preclinical models where resistance to VEGF blockade is shown to induce hypoxia which, in turn, gives rise to an increasing regulation of other pro-angiogenic factors, such as FGF, that continue to stimulate the process of tumour neo-angiogenesis (3). These results are just a tiny sample of what now emerges as a complex puzzle. The process of tumour neo-angiogenesis is a complex biological process involving many cell types and multiple molecular signalling pathways (VEGF, PDGF, TGF-beta, FGF, ANG/TIE, NOTCH/WNT, integrins, chemokines amongst others) that specifically interact in space and time [4]. It is simply naive to expect that the blockade of a single molecule-such as VEGF, despite its major role in the process of angiogenesis-would be capable of completely inhibiting this biological process [5].

Even so, in spite of the complexity involved, treatment with bevacizumab does have an impact on the natural history of the disease. Even though the effect on PFS and overall survival is modest or non-existent, we must not overlook the unequivocal 10-20\% increase in response rates [1] or the clinical observations that long-lasting PFS periods of undeniable clinical value have been achieved in a certain group of patients. So, how can we harness this enormous biological complexity with optimisation of antiangiogenic therapy in clinical practice?

We probably need several different approaches and, perhaps paradoxically, we need both less and more empiricism to address this issue. By less empiricism, I am refer- 
ring to conducting clinical trials based on the plethora of preclinical knowledge currently available. It is disheartening to see that clinical trials on anti-angiogenic drugs have indiscriminately copied patterns used in the past to develop classic chemotherapy with the mere addition of bevacizumab or TKIs to the usual chemotherapy regimens used in the disease. Such trials have ignored concepts based on well established animal models, such as the special synergy dependent on how the different anti-angiogenic drugs are administered alone or combined with chemotherapy [6, 7] or the concept of vascular normalisation [8]. By more empiricism, I mean that we are urgently in need of predictive factors for the efficacy of these therapies [9]. Currently, the absence of predictive factors stands as a major barrier to optimisation of these drugs in clinical practice. Given the complexity of the process and the absence of a complete understanding of all the factors involved [4], we may need to resort to empirical methods (essentially non-biological approaches), such as the massive analysis of data from multiple sources (genomic, clinical, radiological, etc.) associated with efficacy and resistance [10]. To be able to optimise anti-angiogenic therapy for our patients, we must combine fast, appropriate experimental evaluation of preclinical knowledge with empirical evaluation of predictive factors. Otherwise, we will be walking down a controversial, comfortable and well beaten track: to nowhere.

\section{References}

1. Goldfarb SB, Hudis C, Dickler MN (2011) Bevacizumab in metastatic breast cancer: when may be used? Ther Adv Med Oncol 3:85-93

2. Trinh XB, van Dam PA, Vermeulen PB et al (2011) VEGF-A-independent and angiogenesisdependent tumour growth in patients with metastatic breast cancer. Clin Trans Oncol 13:805-808

3. Casanovas O, Hicklin D, Bergers G et al (2005) Drug resistance by evasion of antiangiogenic targeting of VEGF signalling in late-stage pancreatic islet tumors. Cancer Cell 8:299-309
4. Carmeliet P, Jain RK (2011) Molecular mechanisms and clinical applications of angiogenesis. Nature 473:298-307

5. Quesada AR, Medina MA, Alba E (2007) Playing only one instrument may be not enough: limitations and future of the antiangiogenic treatment of cancer. Bioessay 29:1159-1168

6. Bergers G, Song S, Meyer-Morse N et al (2003) Benefits of targeting both pericytes and endothelial cells in the tumor vasculature with kinase inhibitors. J Clin Invest 111:1287-1295

7. Pietras K, Hanahan D (2005) A multitargeted, metronomic, and maximum-tolerated dose chemo- switch regimen is antiangiogenic producing objective responses and survival benefit in a mouse model of cancer. J Clin Oncol 23:939-952

8. Jain RK (2005) Normalization of tumor vasculature: an emerging concept in antiangiogenic therapy. Science 307:58-62

9. Jubb AM, Harris AL (2010) Biomarkers to predict the clinical efficacy of bevacizumab in cancer. Lancet Oncol 11:1172-1183

10. Prasasya RD, Tian D, Kreeger PK (2011) Analysis of cancer signalling networks by systems biology to develop therapies. Sem Cancer Biol 21:200206 